\title{
Photoelectron Spectroscopy of Molecules Beyond the Electric Dipole Approximation
}

\author{
Iulia Emilia Brumboiu, ${ }^{*}, \dot{\dagger}, \odot$ Olle Eriksson, ${ }^{\|, \S}$ and Patrick Norman ${ }^{\dagger \odot}$ \\ ${ }^{\dagger}$ Department of Theoretical Chemistry and Biology, KTH Royal Institute of Technology, 10691 Stockholm, Sweden \\ ${ }^{\ddagger}$ Department of Chemistry, Korea Advanced Institute of Science and Technology, 34141 Daejeon, Korea \\ "Department of Physics and Astronomy, Uppsala University, 75120 Uppsala, Sweden \\ ${ }^{\S}$ School of Science and Technology, Örebro University, 70182 Örebro, Sweden
}

Supporting Information

\begin{abstract}
A methodology implemented to compute photoionization cross sections beyond the electric dipole approximation using Gaussian type orbitals for the initial state and plane waves for the final state is applied to molecules of various sizes. The molecular photoionization cross sections computed for valence molecular orbitals as a function of photon energy present oscillations due to the wave-like nature of both the outgoing photoelectron and of the incoming photon. These oscillations are damped by rotational and vibrational averaging or by performing a k-point summation for the solid state case. For core orbitals, the corrections

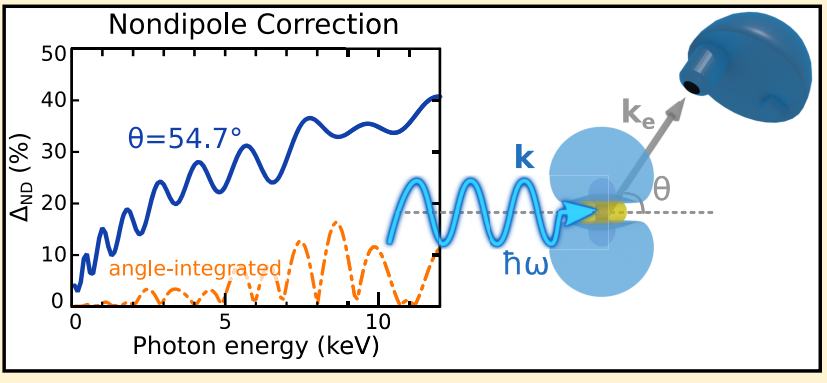
introduced by going beyond the electric dipole approximation are comparable to the atomic case. For valence orbitals, nondipole corrections to the total photoinization cross sections can reach up to $20 \%$ at photon energies above $1 \mathrm{keV}$. The corrections to the differential cross sections calculated at the magic angle are larger, reaching values between $30 \%$ and $50 \%$ for all molecules included. Our findings demonstrate that photoelectron spectroscopy, especially angle-resolved, on, e.g., molecules and clusters on surfaces, using high photon energies, must be accompanied by theories that go beyond the electric dipole approximation.
\end{abstract}

\section{INTRODUCTION}

Photoelectron (PE) spectroscopy, known also as photoemission in the solid state community, is a characterization technique which probes the occupied electronic structure of materials by measuring the kinetic energy of electrons emitted upon photoionization. Considering that occupied electronic states with different atomic contributions have different probabilities of ionization depending on the energy of the ionizing radiation, PE spectra measured at different photon energies provide insight into the contribution of different atomic states to molecular orbitals (MOs) or electronic energy bands. ${ }^{1,2}$ These insights are relevant, for example, in the field of molecular electronics and spintronics, where the nature of the frontier orbitals plays an important role on the functioning of devices. ${ }^{3,4}$

From a computational perspective, the comparison between calculated and measured PE spectra is widely used to asses the quality of a particular electronic structure method. ${ }^{5,6}$ Usually, the computed electronic structure is directly compared to PE spectra measured at low photon energies. References 7-18 constitute several examples of this practice applied at different levels of theory, from density functional theory (DFT) and the $G W$ approximation to Dynamical Mean Field Theory (DMFT).
The inclusion of photoionization cross sections in constructing theoretical PE spectra becomes important for materials composed of metal atoms and their ligands, such as organo-metallics, transition metal complexes, and transition metal oxides. In these cases, the photoionization probability of metal states differs substantially from the photoionization probability of ligand states, especially at larger photon energies. ${ }^{1,2}$ For these types of materials, photoionization cross sections can be taken into account in the calculated PE spectrum either by assuming a simple Gelius model ${ }^{2}$ and multiplying each projected density of states (PDOS) with the corresponding atomic photoionization cross section or by computing the actual photoionization cross section for each particular state. The former method, although very simple, has been quite successful in reproducing valence PE spectra of reasonably large organic molecules with metal atom centers $^{19-22}$ or of periodic transition metal oxides, ${ }^{23,24}$ by making use of either calculated or tabulated dipole atomic photoionization cross sections. ${ }^{25-27}$ The later method involves the direct calculation of transition matrix elements between occupied molecular orbitals and a suitably chosen final state. In

Received: May 14, 2019

Published: September 11, 2019 
contrast to the atomic case, where the spherical symmetry of the effective Coulomb potential allows computing rather accurate wave functions for the continuum states, molecular systems are more challenging. Within the electric dipole (ED) approximation, equations for computing molecular photoionization cross sections have been implemented for different types of initial and final state wave functions. Dyson orbitals have been successfully employed in combination with plane waves (PWs), ${ }^{28-31}$ Coulomb waves, ${ }^{28}$ or B-splines ${ }^{32}$ to compute photoionization cross sections of organic molecules and molecular ions. Another approach has been to use Bsplines to describe both initial and continuum states and compute dipole photoionization cross sections of a series of small molecules. ${ }^{3-36}$ For solid state materials, time-reversed low energy electron diffraction (LEED) states have been successfully used to compute angle-resolved photoemission spectra (ARPES). ${ }^{37-42}$

Besides dipole photoionization cross sections, another important role in the theory-experiment comparison may be played by nondipole (ND) effects, especially at large photon energies and for delocalized states. The results by Panda et al. $^{23}$ constitute one example of theory-experiment differences which could be explained by the fact that ND effects were not included in the calculation. In ref 23 , the spectrum calculated for $\mathrm{NiO}$ with an accurate electronic structure method matches well the experimental valence band PE spectrum at low photon energy $(2 \mathrm{keV})$, but the theory-experiment comparison becomes gradually worse at higher photon energies (4 and 6 $\mathrm{keV}$ ). Since ND effects are expected to increase with photon energy, they could explain why the differences between theory and experiment become larger as the photon energy is increased. The efforts in ref 23 represent a high kinetic energy (HIKE) approach that has the obvious advantages of being less surface-sensitive and, hence, yielding better bulk-like features. However, a problem with HIKE measurements is concerned with the interpretation as it is less than obvious how relevant the electric-dipole approximation is.

In the case of atomic photoionization, there are many studies that go beyond the electric dipole approximation, and we refer the reader to refs 43-52 for different perspectives and overviews. ND corrections to the photoionization cross sections of atomic orbitals increase linearly with photon energy and are quite small, reaching up to $5-10 \%$ at a photon energy of $12 \mathrm{keV}^{53}$

In the case of molecules, there are several experimental studies which investigate nondipole effects in molecular photoionization by determining photoelectron angular distributions and nondipole asymmetry parameters. Among the studied molecules we note $\mathrm{H}_{2},{ }^{54} \mathrm{~N}_{2},{ }^{55,56} \mathrm{~N}_{2} \mathrm{O},{ }^{55} \mathrm{CO},{ }^{56} \mathrm{Br}_{2}$, and $\mathrm{BrCF}_{3}{ }^{57}$

When it comes to theoretical studies, first order corrections to the electric dipole approximation have been implemented at several levels of theory and applied to compute nondipole photoelectron angular distributions and asymmetry parameters. In particular, the random phase approximation (RPA) has been applied to determine nondipole effects in the photoionization of $\mathrm{N}_{2}{ }^{58}$ and $\mathrm{CO} .{ }^{56}$ At the DFT level of theory, we mention the implementation of Toffoli and Decleva using Bsplines, ${ }^{59,60}$ which has been employed to compute the nondipole asymmetry parameters of $\mathrm{N}_{2},{ }^{61} \mathrm{CF}_{4},{ }^{62} \mathrm{SF}_{6},{ }^{59}$ and $\mathrm{C}_{60}{ }^{63}$ at photon energies up to $200 \mathrm{eV}$ above the ionization threshold. Finally, we mention the work of Seabra et al. ${ }^{64}$ who calculated ND differential cross sections and corrections for photon energies up to $5 \mathrm{keV}$ for four small molecules (HF, $\mathrm{H}_{2} \mathrm{O}, \mathrm{NH}_{3}$, and $\mathrm{CH}_{4}$ ), using Dyson orbitals for the initial state and a PW for the final state. In ref 64, the full field operator has been used to determine the magnitude of ND corrections to molecular photoionization, which were found to reach up to $40 \%$.

In the present study, we aim to estimate the magnitude of $\mathrm{ND}$ corrections for molecules of various sizes, including large systems such as $\mathrm{C}_{60}$. We apply a previously developed methodology ${ }^{53}$ based on the expression beyond the dipole approximation obtained by List et al. for the photoabsorption cross section. ${ }^{65}$ Using Gaussian type orbitals (GTOs) for the initial state and PWs for the final state, we compute the dipole and nondipole photoionization cross sections as a function of photon energy up to $12 \mathrm{keV}$ for a set of 17 molecules of various sizes, from diatomic molecules to $\mathrm{C}_{60}$. We find that, overall, the corrections to the dipole approximation can be very large, especially for high photon energies and angle-resolved cases. Hence investigations that aim to obtain information both on bulk and surface (e.g., adsorbed molecules or atoms) in the same experiment, where high kinetic energy is required, must be accompanied by a theory that goes beyond the electric dipole approximation.

\section{THEORY}

Starting from the expression for the photoabsorption cross section derived by List and co-workers: ${ }^{65}$

$$
\lim _{\left\{\gamma_{n}\right\} \rightarrow 0} \sigma(\omega)=\frac{\pi \hbar e^{2}}{\varepsilon_{0} c \omega m_{\mathrm{e}}^{2}} \sum_{n>0}\left|\left\langle 0\left|\sum_{j=1}^{N} e^{-i \mathbf{k} \cdot \mathbf{r}_{j}} \epsilon \cdot \nabla_{j}\right| n\right\rangle\right|^{2} \delta\left(\omega_{n 0}-\omega\right)
$$

we obtain an equation for the photoionization cross section where the final state of the photoelectron is described as a $\mathrm{PW}^{53}$

In eq $1, \hbar, e, \varepsilon_{0}, c$, and $m_{e}$ are the reduced Planck constant, the elementary charge, the vacuum permittivity, the light velocity in vacuum, and the electron mass, respectively; $\omega$ is the angular frequency of the electromagnetic field; the summation is over all many-body final states $|n\rangle$, and the nondipole transition matrix element is computed between the ground state $|0\rangle$ and the final state $|n\rangle ;\left\{\gamma_{n}\right\}$ are the relaxation parameters for the set of excited states and which correspond to their inverse finite lifetimes; $\mathbf{k}$ represents the wave vector corresponding to the electromagnetic field; $\mathbf{r}_{j}$ is the position vector of electron $j ; N$ is the total number of electrons; $\epsilon$ is the polarization direction; and $\omega_{n 0}$ is the transition angular frequency corresponding to the energy difference between the final and initial states.

Employing a frozen orbital approximation as described in ref 53 and replacing the single particle final state by a PW with wave vector $\mathbf{k}_{\mathrm{e}}$, the expression for the photoionization cross section becomes ${ }^{53}$

$$
\lim _{\gamma_{f} \rightarrow 0} \sigma(\omega)=\frac{e^{2}}{\pi \varepsilon_{0} c m_{e}} \frac{k_{e}}{\omega}\left|\epsilon \cdot \mathbf{k}_{\mathrm{e}}\left\langle\chi_{i} \mid e^{i \mathbf{k}_{\mathrm{e}} \cdot \mathbf{r}-i \mathbf{k} \cdot \mathbf{r}}\right\rangle\right|^{2}
$$

where we here consider a transition from the ground state to a specific final state $|f\rangle$ that is characterized by an electronic transition from occupied orbital $\chi_{i}$ to a free-electron state. As a result, the index $j$ has been dropped when moving from a many-body description to using single electron states, and initial state $\chi_{i}$ is an occupied molecular orbital expressed as a 
linear combination of atomic orbitals (AO), denoted $\phi_{\mu}$. Representing further each $\mathrm{AO}$ as a linear combination of GTOs, the final expression for the photoionization cross section becomes ${ }^{53}$

$$
\lim _{\gamma_{f} \rightarrow 0} \sigma(\omega)=\frac{e^{2}}{\pi \varepsilon_{0} c m_{e}} \frac{k_{e}}{\omega}\left|\epsilon \cdot \mathbf{k}_{\mathrm{e}} \sum_{\mu} c_{\mu} \sum_{l} d_{\mu l}\left\langle g_{\mu l} \mid e^{i \mathbf{k}_{\mathrm{e}} \cdot \mathbf{r}-i \mathbf{k} \cdot \mathbf{r}}\right\rangle\right|^{2}
$$

where $c_{\mu}$ is the coefficient of $\phi_{\mu}$ in the linear combination, the first summation $(\mu)$ is over all atomic orbitals which contribute to the ionized molecular orbital, and the second summation $(l)$ is over all primitives used to describe atomic orbital $\phi_{\mu}$, where $g_{\mu L}$ is a GTO with $l=l_{x}+l_{y}+l_{z}{ }^{53}$

The dipole approximation is straightforwardly retrieved by setting $\mathbf{k}$ to zero in eq 3 . We note that by using the full field operator in eq 3 , the photoionization cross sections are gauge origin independent. $^{65,66}$

\section{COMPUTATIONAL DETAILS}

The ground state structures of the molecules in Figure 1 were optimized with use of the Gaussian 16 quantum chemistry

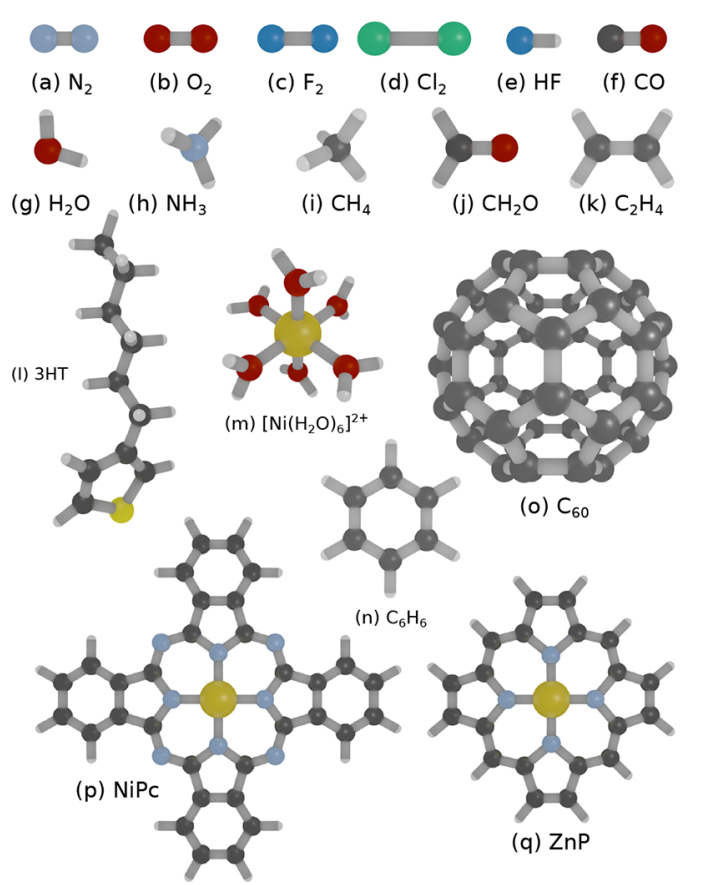

Figure 1. Molecules studied in the present work: (a) nitrogen, (b) oxygen, (c) fluorine, (d) chlorine, (e) hydrofluoric acid, (f) carbon monoxide, (g) water, (h) ammonia, (i) methane, (j) formaldehyde, (k) ethene, (l) 3-hexylthiophene, (m) hexaaquanickel(II) ion, (n) benzene, $(\mathrm{o}) \mathrm{C}_{60}$ fullerene, $(\mathrm{p})$ nickel phthalocyanine, and ( $\mathrm{q}$ ) zinc porphyrin.

software ${ }^{67}$ using the B3LYP ${ }^{68}$ functional in combination with the universal Gaussian basis set (UGBS) by de Castro et al. $^{69-77}$ in its Cartesian Gaussians variant. The ground state Kohn-Sham (KS) molecular orbitals were used in eq 3 to compute rotationally averaged photoionization cross sections within and beyond the electric dipole approximation. These cross sections were determined for each molecule using the computational setup in Figure $2 b$, where the photon wave vector $(\mathbf{k})$ was rotated to directions generated by the Lebedev quadrature of order $n=194 .^{78}$ For each configuration, the integral over the polarization direction was performed as described in ref 66, by representing the transition moment in the photon reference frame, with the photon wave vector on the $z$-axis and the polarization direction in the $x y$-plane. In this reference frame, the direction of the photoelectron wave vector was generated via the Lebedev quadarture of order $n=194$, while its norm $k_{\mathrm{e}}$ was determined from energy conservation. The total photoionization cross section was obtained by taking the average of all configurations $(194 \times 194)$ weighted by their corresponding Lebedev weights. The cross section calculated this way corresponds to performing angle-integrated photoelectron spectroscopy measurements of a gas-phase system, and it will be referred to as rotationally averaged cross section throughout this work.

In addition, a differential cross section was calculated with the same procedure but keeping the angle between the photon and photoelectron wave vectors fixed at $\theta=54.7^{\circ}$ (magic angle, MA). In this case, the photoelectron direction was not generated by the Lebedev quadrature but by rotating $k_{\mathrm{e}}$ around $\mathbf{k}$ by $360^{\circ}$ with a step of $5^{\circ}$. This corresponds to a gas-phase photoionization experiment where the detector is placed at $\theta=$ $54.7^{\circ}$ with respect to the photon beam. The cross section computed with this procedure will be referred to as the differential cross section.

In the case of the $\mathrm{N}_{2}$ molecule, a vibrationally averaged cross section was computed as follows: (1) The vibrational frequency of the $\mathrm{N}-\mathrm{N}$ stretching mode was determined at the B3LYP ${ }^{68}$ level of theory and using the UGBS basis set. $^{69-77}$ (2) Several configurations (denoted i) of the $\mathrm{N}_{2}$ molecule were obtained by stretching or contracting the $\mathrm{N}-\mathrm{N}$ bond from its calculated equilibrium value of $1.109 \AA$. The specific bond lengths considered were $Q_{i}=0.986,1.008,1.030$, $1.052,1.074,1.096,1.118,1.140,1.162,1.184$, and $1.206 \AA$. (3) The $\mathrm{ED}$ and, respectively, ND photoionization cross sections $\left(\sigma_{i}\right)$ were calculated for each configuration $i$ using the computational setup of Figure 2c. (4) The vibrationally averaged value of the cross section was obtained by weighing each $\sigma_{i}$ with the probability density $\left|\psi_{s}\left(Q_{i}\right)\right|^{2}$, where $\psi_{s}\left(Q_{i}\right)$ is the value of the nuclear wave function corresponding to the $\mathrm{N}-\mathrm{N}$ stretching vibration at the $\mathrm{N}-\mathrm{N}$ bond length $Q_{i}$.

Finally, to model the effects of a periodic crystal, a k-point summation was performed in the case of the $\left[\mathrm{Ni}\left(\mathrm{H}_{2} \mathrm{O}\right)_{6}\right]^{2+}$ molecular ion, which represents a molecular model for the $\mathrm{NiO}$ crystal, since the chemical building block of bulk $\mathrm{NiO}$ is an octahedron of $\mathrm{O}$ atoms with a $\mathrm{Ni}$ atom in the center. The nominal charge of bulk $\mathrm{NiO}$ is $\mathrm{Ni}^{2+}$ and $\mathrm{O}^{2-}$, which is captured by the $\mathrm{Ni}$ and $\mathrm{O}$ atoms in $\left[\mathrm{Ni}_{(}\left(\mathrm{H}_{2} \mathrm{O}\right)_{6}\right]^{2+}$. The k-point summation was performed by constructing a k-dependent electronic wave function $\psi_{k_{z}}$ for each molecular orbital $\chi_{i}$ determined with the B3LYP functional and the UGBS basis set:

$$
\psi_{k}(\mathbf{r})=\sum_{\mu=1}^{\mathrm{NAO}} c_{\mu i} e^{i k_{z} A_{z}} \phi_{\mu}(\mathbf{r}-\mathbf{R})
$$

where $\phi_{\mu}$ is the $\mu$ th atomic orbital centered at the atom site $\mathbf{R}$, $c_{\mu i}$ represents the contribution of $\phi_{\mu}$ to molecular orbital $\chi_{i}$, and $A_{z}$ is the $z$ coordinate of $\mathbf{R}$. The index $z$ indicates that only periodicity in the $z$-direction was considered. This approximate treatment of the $\mathrm{NiO}$ band structure was made to illustrate the importance of nondipolar effects of the angular integrated 
(a)

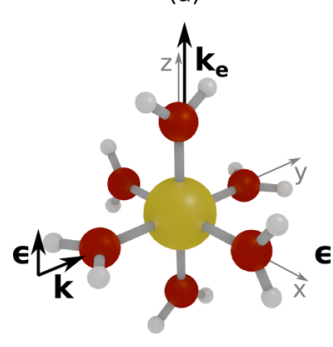

(b)

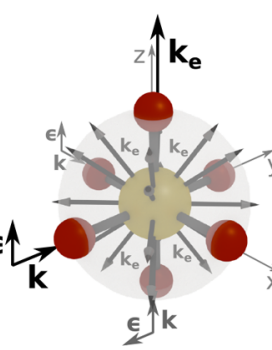

(c)



(d)

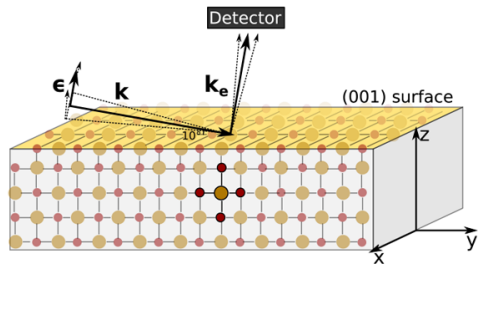

Figure 2. (a) Fixed computational setup used to compute the dipole and nondipole photoionization cross sections of $\left[\mathrm{Ni}\left(\mathrm{H}_{2} \mathrm{O}\right)_{6}\right]^{2+}$. $(\mathrm{b})$ Computational setup for rotational averaging. The $\mathbf{k}$ wave vector is rotated to directions generated via the Lebedev quadrature. Each configuration is then combined with photoelectron directions generated via the Lebedev quadrature in the photon reference frame. The arrows mark several directions generated for the $\mathbf{k}_{\mathbf{e}}$ wave vector obtained at order $n=14$ of the quadrature. (c) Computational setup for vibrational averaging. (d) Illustration of the $\mathrm{NiO}$ crystal, alongside an experimental setup to measure photoemission. Red and yellow spheres symbolize $\mathrm{O}$ and $\mathrm{Ni}$ atoms, respectively.

photoemission experiments of solids and how k-space summation influence the calculated cross sections.

By replacing $\chi_{i}$ in eq 2 with $\psi_{k}$ from eq 4 , a photoionization cross section was determined using the computational setup from Figure $2 \mathrm{a}$ for each $k_{z}$ in the interval $[-2 \pi / a, 2 \pi / a]$ which corresponds to the first Brillouin zone of a face-centered cubic lattice. Here, $a=4.143 \AA$ represents the $\mathrm{NiO}$ lattice constant, i.e., $2 \times$ the B3LYP calculated $\mathrm{Ni}-\mathrm{O}$ bond length in the $\left[\mathrm{Ni}\left(\mathrm{H}_{2} \mathrm{O}\right)_{6}\right]^{2+}$ complex. Considering that $\mathrm{NiO}$ is an insulator, all occupied bands are below the Fermi level and do not cross it, so the k-point summation must be performed over the full interval. However, to qualitatively asses the effects of k-point summation, we additionally tested two other smaller intervals, $[-\pi / a, \pi / a]$ and, respectively, $[-\pi / 2 a, \pi / 2 a]$. The k-point summation was performed by generating 100 equidistant $k_{z}$ values in the given interval and calculating a cross section for each of them. The final value of the photoionization cross section was determined as the sum of the cross sections obtained for each $k_{z}$, divided by the number of k-points included.

Finally, for all molecules included in this study, ND corrections were computed from the rotationally averaged and, respectively, differential dipole $\left(\sigma_{\mathrm{ED}}\right)$ and nondipole $\left(\sigma_{\mathrm{ND}}\right)$ cross sections using the following equation:

$$
\Delta_{\mathrm{ND}}=100 \frac{\left|\sigma_{\mathrm{ND}}-\sigma_{\mathrm{ED}}\right|}{\sigma_{\mathrm{ND}}}(\%)
$$

All cross sections and corrections were calculated for the photon energy range between $10 \mathrm{eV}$ and $12 \mathrm{keV}$.

\section{RESULTS AND DISCUSSION}

In the following, the features of the photoionization cross sections as a function of photon energy for the core and valence molecular orbitals of the $\mathrm{N}_{2}$ molecule are thoroughly analyzed. The oscillations observed in the cross sections are explained, alongside several damping mechanisms, where the $\mathrm{k}$ point summation is performed for the $\left[\mathrm{Ni}\left(\mathrm{H}_{2} \mathrm{O}\right)_{6}\right]^{2+}$ molecular ion. The magnitude of the ND corrections to the total and differential cross sections is reported for all included molecules in several photon energy windows.

We begin by discussing the electronic structure of the $\mathrm{N}_{2}$ molecule since it will be relevant in explaining the behavior of the photoionization cross sections as a function of photon energy. For completeness, we depict in Figure 3 a schematic representation of the well-known electronic levels of $\mathrm{N}_{2}$ with (a) MO diagram

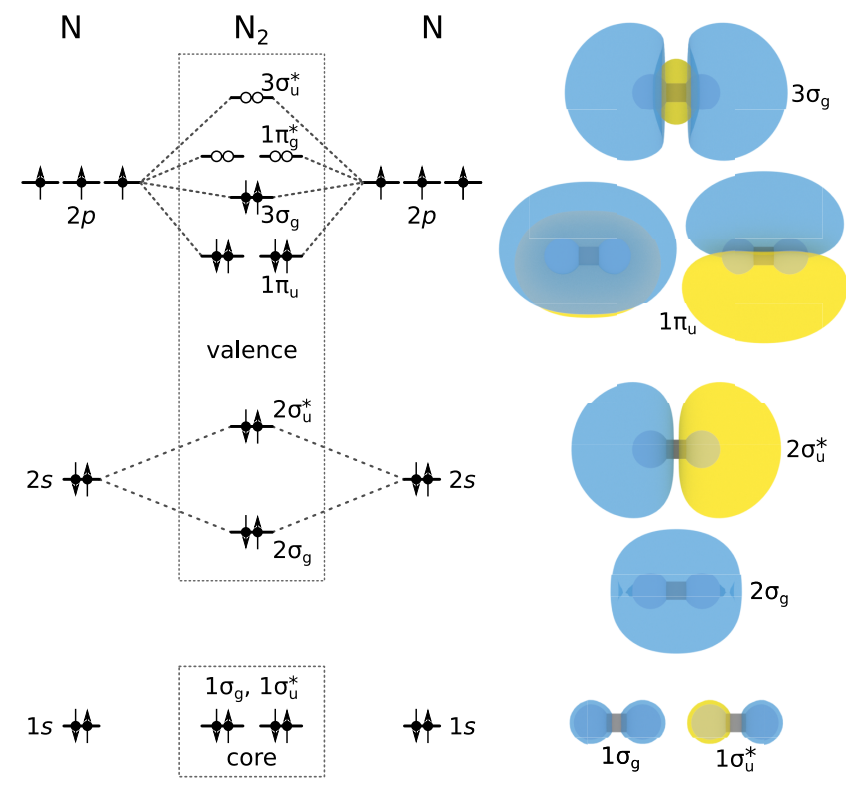

Figure 3. (a) Molecular orbital diagram for the $\mathrm{N}_{2}$ molecule (not to scale). The antibonding molecular orbitals are marked with an asterisk. (b) Illustration of the occupied molecular orbitals of $\mathrm{N}_{2}$ represented using a wave function iso-surface absolute value of 0.02 au. Positive surfaces are represented in blue, while negative surfaces are in yellow.

hybridized atomic orbitals forming bonding and antibonding molecular orbitals. In the case of the core levels, the atomic 1s wave functions give rise to a pair of almost degenerate MOs (separated by only $\sim 0.05 \mathrm{eV}$ in our calculation), denoted $1 \sigma_{\mathrm{g}}$ and $1 \sigma_{\mathrm{u}}^{*}$ in Figure 3, where the asterisk marks the antibonding MO. In the case of the valence, the $2 \mathrm{~s}$ AOs hybridize to form the nondegenerate MOs (separated by $\sim 17 \mathrm{eV}$ in our calculation) $2 \sigma_{\mathrm{g}}$ and $2 \sigma_{\mathrm{u}}^{*}$ which are both occupied, while the $2 \mathrm{p}$ AOs result in partially nondegenerate MOs where only the bonding counterparts are occupied.

Photoionization of Core Levels. Figure $4 \mathrm{a}$ shows the dipole photoionization cross section as a function of photon energy calculated for the bonding (blue) and antibonding (orange) core (1s) MOs of $\mathrm{N}_{2}$. The setup used for the calculations is depicted in the inset, with the $\mathrm{N}-\mathrm{N}$ bond placed parallel to the $z$-axis, the photon wave vector in the $y$ - 
(a)

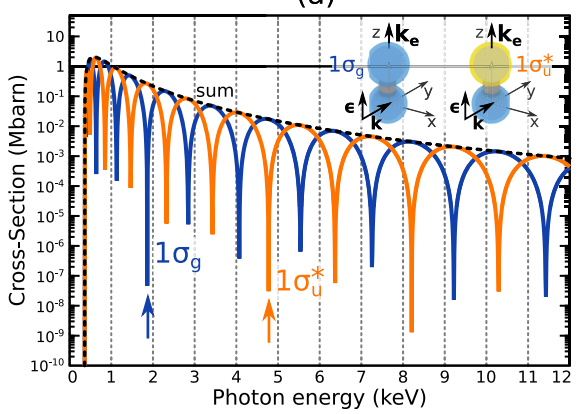

(b)

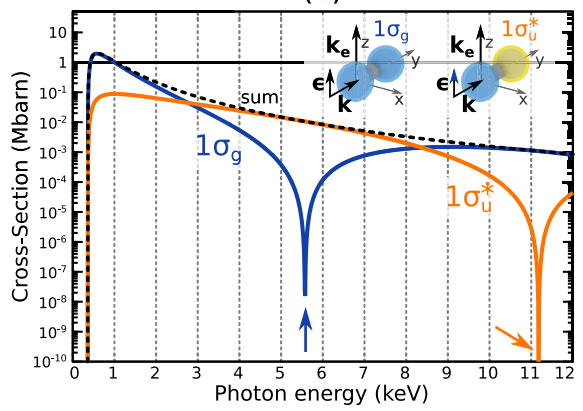

(c)

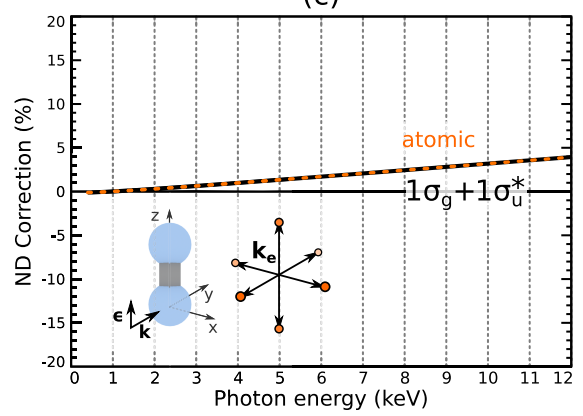

Figure 4. (a) Calculated ED cross sections of the 1s MOs of the $\mathrm{N}_{2}$ molecule in a fixed setup, depicted in the inset, where the molecule is parallel to the photoelectron wave vector. (b) Calculated ND cross section of the 1s MOs of the $\mathrm{N}_{2}$ molecule in a fixed setup, depicted in the inset, where the molecule is parallel to the photon wave vector. (c) ND correction for the $\mathrm{N} 1 \mathrm{~s}$ states in the $\mathrm{N}_{2}$ molecule (black solid line) compared to the $\mathrm{N}$ atom (dotted orange line) calculated using ED and ND cross sections averaged over possible photoelectron directions as performed in ref 53.

direction, and the photoelectron wave vector in the $z$-direction, parallel to the polarization direction.

As the figure shows, the individual cross sections for the two core orbitals present oscillations as a function of photon energy. The photon energies corresponding to the minima of the antibonding $\mathrm{MO} 1 \sigma_{\mathrm{u}}^{*}$ are listed in Table 1 . These minima

Table 1. Photon Energy $(\hbar \omega)$ Positions of the Minima Observed in the ED Photoionization Cross Sections of the Antibonding $1 \sigma_{\mathrm{u}}^{*}$ Molecular Orbital (Figure 4a), alongside the Kinetic Energy (KE), Wave Vector $\left(k_{\mathrm{e}}\right)$, and Wavelength $\left(\lambda_{\mathrm{e}}\right)$ Corresponding to the Photoelectron ${ }^{a}$

$\begin{array}{rcccc}\hbar \omega(\mathrm{keV}) & \mathrm{KE}(\mathrm{keV}) & k_{e}\left(\AA^{-1}\right) & \lambda_{e}(\AA) & n \\ 0.52 & 0.13 & 5.84 & 1.076 & 1.03 \\ 0.88 & 0.49 & 11.34 & 0.554 & 2.00 \\ 1.49 & 1.10 & 16.99 & 0.370 & 3.00 \\ 2.35 & 1.96 & 22.68 & 0.277 & 4.00 \\ 3.45 & 3.06 & 28.34 & 0.222 & 5.00 \\ 4.79 & 4.40 & 33.98 & 0.185 & 5.99 \\ 6.38 & 5.99 & 39.65 & 0.158 & 7.02 \\ 8.21 & 7.82 & 45.30 & 0.139 & 7.98 \\ 10.29 & 9.90 & 50.97 & 0.123 & 9.02\end{array}$

${ }^{a}$ The last column is obtained by dividing the $\mathrm{N}-\mathrm{N}$ equilibrium bond length $(1.109 \AA)$ with $\lambda_{\mathrm{e}}$.

occur when the wavelength corresponding to the photoelectron fits an integer number of times the distance between the two $\mathrm{N}$ atoms. In the case of the bonding orbital $1 \sigma_{\mathrm{g}}$, the minima are shifted by half a period with respect to the minima of $1 \sigma_{\mathrm{u}}^{*}$, as will be shown more rigorously in the following. As a result, the total cross section (dashed line in Figure 4a,b) for the core MOs, obtained as the sum of the bonding and antibonding counterparts, does not present any oscillations and is basically identical to the $\mathrm{N}$ 1s atomic photoionization cross section. ${ }^{53}$

To further explain the oscillatory behavior of the cross sections in Figure 4a, we assume a simplified description of the $1 \sigma_{\mathrm{u}}^{*}$ molecular orbital as a linear combination of two 1s GTOs, each centered on one of the $\mathrm{N}$ atoms in the molecule:

$$
1 \sigma_{\mathrm{u}}^{*}=c e^{-\alpha\left(x^{2}+y^{2}+z^{2}\right)}-c e^{-\alpha\left[x^{2}+y^{2}+(z-b)^{2}\right]}
$$

where $c$ is a linear combination coefficient which we assume, for simplicity, includes the normalization constant, $\alpha$ is the orbital exponent defining the GTO, and $b$ represents the $\mathrm{N}-\mathrm{N}$ bond length. To simplify the expression, we have considered that one of the $\mathrm{N}$ atoms is placed at the origin, while the other is at coordinate $b$ on the $z$-axis.

To compute the ED photoionization cross section for this molecular orbital, we must compute transition matrix elements of the type:

$$
\left\langle 1 \sigma_{\mathrm{u}}^{*} \mid e^{i k_{e} z}\right\rangle=c\left\langle e^{-\alpha\left(x^{2}+y^{2}+z^{2}\right)} \mid e^{i k_{\varepsilon} z}\right\rangle-c\left\langle e^{-\alpha\left[x^{2}+y^{2}+(z-b)^{2}\right]} \mid e^{i k_{e} z}\right\rangle
$$

where we have set the photon wave vector $k=0$ and considered the computational setup in the inset of Figure $4 a$, where the photoelectron wave vector is in the $z$-direction.

The transition matrix elements from eq 7 involve integrals which can be computed analytically, as shown in the Supporting Information of ref 53. Applying the appropriate formulas, the transition matrix element becomes

$$
\begin{aligned}
\left\langle 1 \sigma_{\mathrm{u}}^{*} \mid e^{i k_{e} z}\right\rangle & =c \frac{\pi}{\alpha}\left(\int_{-\infty}^{\infty} e^{-\alpha z+i k_{e} z} \mathrm{~d} z-\int_{-\infty}^{\infty} e^{-\alpha(z+b)^{2}+i k_{e} z} \mathrm{~d} z\right) \\
& =c\left(\frac{\pi}{\alpha}\right)^{3 / 2} e^{-k_{e}^{2} /(4 \alpha)}\left(1-e^{i k_{e} b}\right)
\end{aligned}
$$

From eq 8 , it becomes clear that the dipole photoionization cross section will have minima at photon energies where $k_{e}$ is such that the term in parentheses is zero:

$$
e^{i k_{e} b}=1 \Leftrightarrow k_{e}=\frac{2 \pi n}{b}
$$

where $n$ is an integer.

The same derivation can be performed for a simplified bonding orbital:

$$
1 \sigma_{\mathrm{g}}=c e^{-\alpha\left(x^{2}+y^{2}+z^{2}\right)}+c e^{-\alpha\left[x^{2}+y^{2}+(z-b)^{2}\right]}
$$

with the final solution:

$$
e^{i k_{e} b}=-1 \Leftrightarrow k_{e}=\frac{2 \pi n}{b}+\frac{\pi}{b}
$$

Comparing eqs 9 and 11 , the positions of the minima for bonding and antibonding orbitals differ by half a period and, therefore, the total cross section obtained as the sum of the two presents no oscillatory behavior.

Figure $4 \mathrm{~b}$ shows the ND cross sections of the same core orbitals, $1 \sigma_{\mathrm{g}}$ and $1 \sigma_{\mathrm{u}}^{*}$, as a function of photon energy. By placing the molecule perpendicular to the photoelectron wave vector, as shown in the inset of Figure $4 b$, the oscillations due 
to the photoelectron are suppressed. In this configuration, the $\mathrm{N}-\mathrm{N}$ bond can be said to be "invisible" from the point of view of the photoelectron. What is meant by this can be clarified using the simplified expressions for the core orbitals from eqs 6 and 10 , modified to account for the fact that the $\mathrm{N}-\mathrm{N}$ bond is now parallel to the $y$-axis. The ED matrix elements computed using these orbitals result in a zero cross section for the antibonding orbital and a nonoscillating cross section identical to the atomic case for the bonding orbital. In the ND case, if the molecule is additionally placed parallel to the photon propagation direction, the minima observed in the photoionization cross section are due solely to the photon wave vector. This is further shown in Table 2 which lists the photon

Table 2. Photon Energy ( $\hbar \omega)$ Positions of the Minima Observed for the ND Photoionization Cross Sections of the $1 \sigma_{\mathrm{g}}$ and $1 \sigma_{\mathrm{u}}^{*}$ Molecular Orbitals (Figure $4 \mathrm{~b}$ ), alongside the Photon Wavelength $(\lambda)^{a}$

$\begin{array}{cccc}\mathrm{MO} & \hbar \omega(\mathrm{keV}) & \lambda(\AA) & n \\ 1 \sigma_{\mathrm{g}} & 5.59 & 2.218 & 0.5 \\ 1 \sigma_{\mathrm{u}}^{*} & 11.17 & 1.110 & 1.00\end{array}$

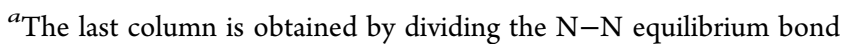
length $(1.109 \AA)$ with $\lambda$.

energy positions of the minima of $1 \sigma_{\mathrm{g}}$ and $1 \sigma_{\mathrm{u}}^{*}$. These photon energies correspond to situations where $k=\pi / b$ (in the case of $1 \sigma_{\mathrm{g}}$ ) and, respectively, $k=2 \pi / b$ (in the case of $1 \sigma_{\mathrm{u}}^{*}$ ).

When the molecule is placed in a random orientation with respect to the $\mathbf{k}_{\mathrm{e}}$ and $\mathbf{k}$ wave vectors, a combination of the two effects discussed above is observed, with minima resulting from the wave-like nature of both the photoelectron and of the photon. In all setups, however, the oscillations vanish once the bonding and antibonding contributions are summed up and the total cross sections, both ED and ND, are identical to the atomic case after taking into account the fact that four electrons occupy the molecular core levels vs two in the atomic case. The ND correction is, therefore, equal to the ND correction obtained for the atom, as illustrated in Figure 4c.

Oscillations in the photoionization cross sections have been first predicted by Cohen and Fano, ${ }^{80}$ who calculated the dipole photoionization cross section of a $\mathrm{H}_{2}$ molecule using Slater type orbitals for the initial state and a free-field spherical wave for the final state and found that coherent photoemission from the two identical centers leads to an interference pattern. In the case of the $\mathrm{N}_{2}$ molecule, the oscillations predicted for the core orbitals by the method employed here are in agreement with the Cohen-Fano (CF) model, ${ }^{79}$ as illustrated in Figure 5. The experimental data shown in Figure 5 were measured using a high resolution soft X-ray spectrometer that could resolve the very small energy difference between the $1 \sigma_{\mathrm{g}}$ and $1 \sigma_{\mathrm{u}}^{*} \mathrm{X}$-ray photoelectron spectroscopy (XPS) peaks. ${ }^{79}$ As shown in the figure, the oscillations observed experimentally are shifted with respect to our results and the CF model. This difference likely arises because of the plane wave (free electron) approximation used to describe the photoelectron wave function. This approximation is especially crude around the photoionization threshold, where the kinetic energy of the photoelectron is not high enough to be able to neglect the Coulomb potential of the ionized atom. As the photon energy and, consequently, the photoelectron kinetic energy is increased, the plane wave approximation becomes more accurate, as discussed in detail for atomic photoionization in ref 53. In the case of molecules

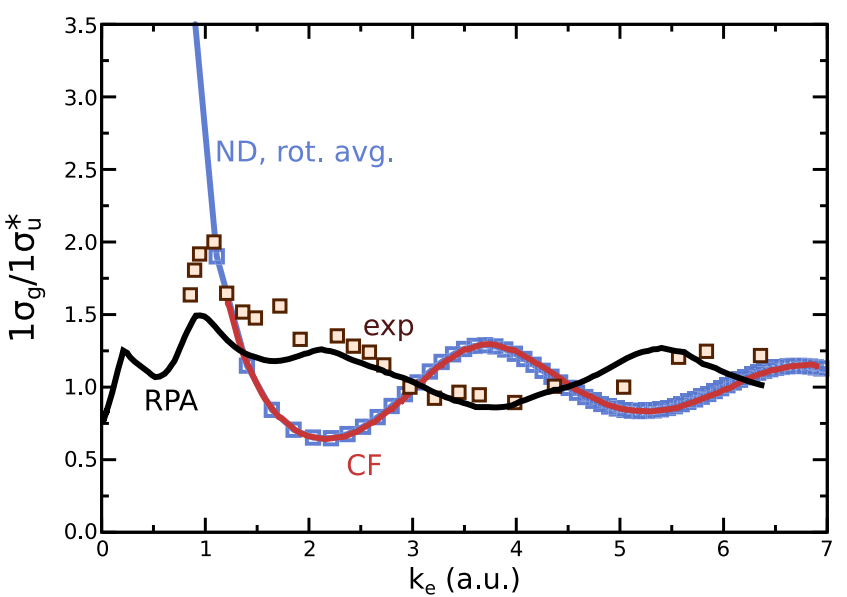

Figure 5. Ratio of the rotationally averaged ND cross sections calculated for the $1 \sigma_{\mathrm{g}}$ and $1 \sigma_{\mathrm{u}}^{*}$ core MOs of $\mathrm{N}_{2}$ (blue line) as a function of the magnitude of the photoelectron wave vector shown in comparison to previous theoretical (red and black lines) and experimental (squares) data from ref 79 . The previous theoretical data is obtained using the interference model proposed by Cohen and Fano (CF) (red) ${ }^{80}$ or using the random phase approximation (RPA, black), including the effect of photoelectron scattering by the neighboring $\mathrm{N}$ atom. ${ }^{79}$

and solids, possible multiple scattering mechanisms may play a role. This is illustrated in Figure 5, where the random phase approximation (RPA) result of Liu et al., ${ }^{79}$ accounting for scattering by the neighboring $\mathrm{N}$ atom, is able to reproduce the experiment. In solids, multiple scattering events must be considered to replicate the experimental extended X-ray absorption fine structure (EXAFS). ${ }^{81-83}$

Photoionization of Valence Levels. In the case of valence molecular orbitals, as anticipated by the description of the $\mathrm{N}_{2}$ electronic structure, the oscillatory behavior of the photoionization cross section persists because the bonding and antibonding MOs are nondegenerate or partially unoccupied, so that their separate contributions cannot be summed up. Figure $6 \mathrm{a}$ shows the calculated ND photoionization cross section for the $3 \sigma_{\mathrm{g}} \mathrm{MO}$ of the $\mathrm{N}_{2}$ molecule, in a fixed setup where the bond-axis of the molecule is parallel to the photoelectron wave vector $\mathbf{k}_{\mathrm{e}}$. Similar to the case of core orbitals, the cross section has minima for photon energies where the $\mathbf{k}_{\mathrm{e}}$ and $\mathbf{k}$ vectors are such as to zero out the transition matrix elements. The analysis is slightly more complicated than in the case of the $1 \mathrm{~s}$ MOs because the $3 \sigma_{\mathrm{g}}$ $\mathrm{MO}$ involves a linear combination of $p$-type GTOs, but the principle is the same.

Oscillations in the intensity of peaks in the valence PE spectra of materials have been experimentally observed notably in the vibrationally resolved spectra of small molecules ${ }^{36}$ and in the gas-phase spectra of $\mathrm{C}_{60}$ and $\mathrm{C}_{70} \cdot{ }^{84,85}$ Rather than vanishing at certain photon energies as our fixed setup model predicts, the relative intensities of the $\mathrm{C}_{60} \mathrm{HOMO}$ and HOMO-1 peaks oscillate around an "equilibrium" ratio, 85 suggesting the existence of certain damping mechanisms. In gas-phase measurements, the molecules are free to rotate and vibrate, while in solid state photoemission experiments, the atoms are part of a periodic crystal. From eqs 9 and 11, it is clear that by altering the distance between the two atoms, as for example during a stretching vibrational mode, the photon energy positions of the cross section minima will shift, resulting in an average cross section with quenched oscillatory behavior. 
(a)



(b)

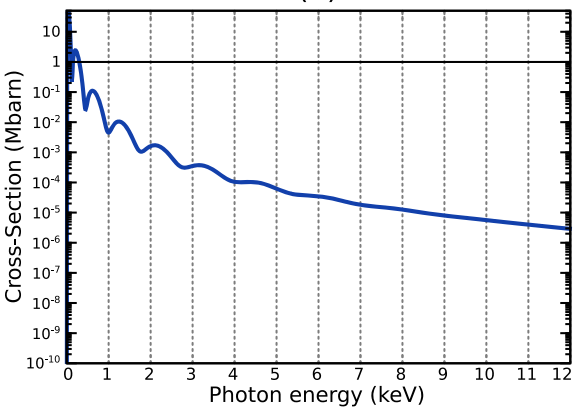

(c)

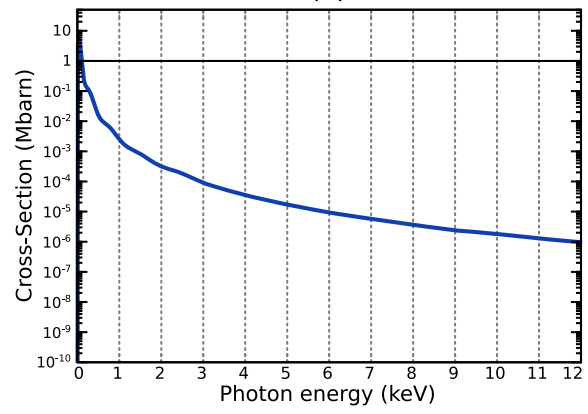

Figure 6. (a) Calculated ND photoionization cross-section for the $3 \sigma_{\mathrm{g}}$ orbital of the $\mathrm{N}_{2}$ molecule in a fixed setup, depicted in the inset, where the molecule is parallel to the photoelectron wave vector. (b) Vibrationally averaged ND cross section of the same orbital, computed for the same fixed setup. (c) Rotationally averaged ND cross section of the $3 \sigma_{\mathrm{g}} \mathrm{MO}$.

(a)

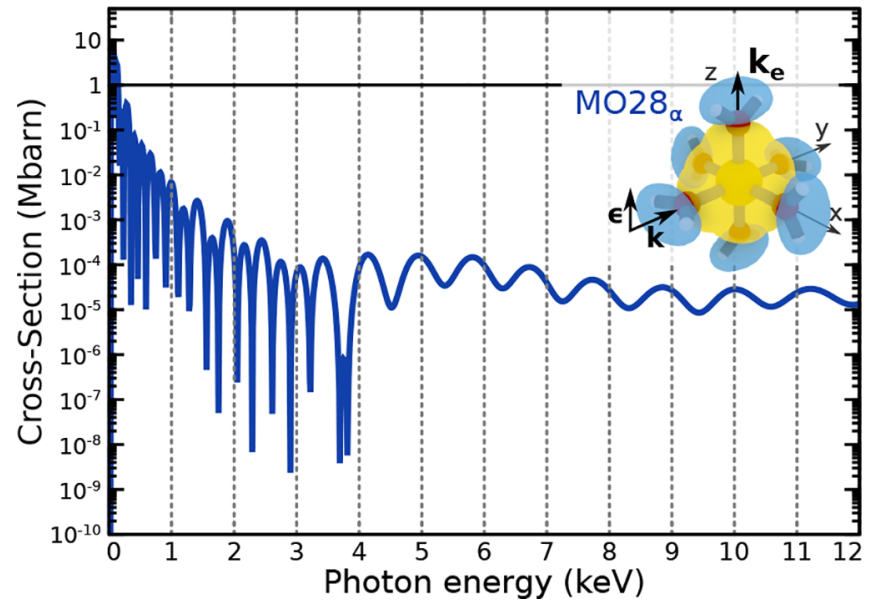

(b)

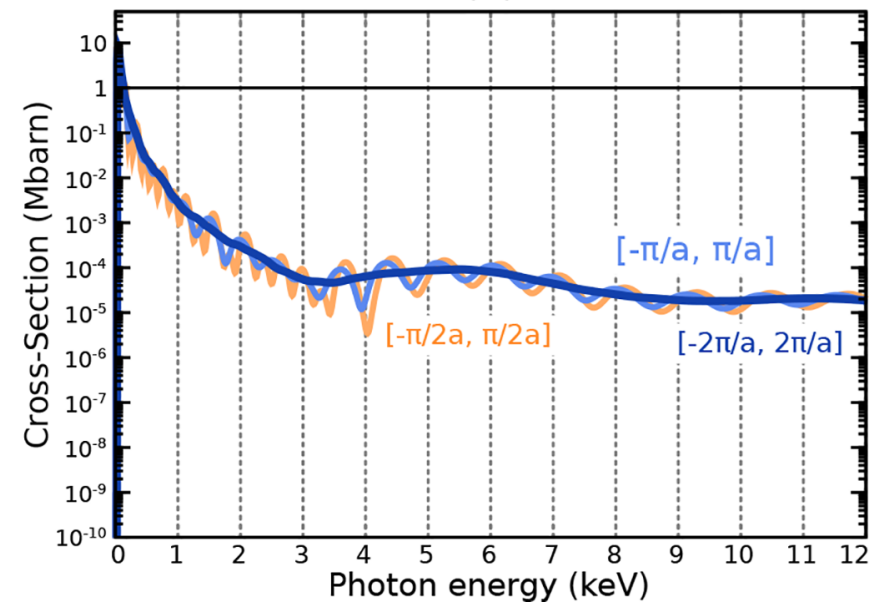

Figure 7. (a) Calculated ND photoionization cross section of one of the molecular orbitals of the $\left[\mathrm{Ni}\left(\mathrm{H}_{2} \mathrm{O}\right)_{6}\right]^{2+}$ complex in a fixed setup, depicted in the inset, where the out-of-plane pair of $\mathrm{Ni}-\mathrm{O}$ bonds is parallel to the photoelectron wave vector. (b) Average of data from Figure $7 \mathrm{a}$, obtained after k-point summation over three different k-point intervals (for details see text). The subscript $\alpha$ indicates that only the spin up orbital is shown.

This is illustrated in Figure $6 \mathrm{~b}$ which depicts the zero point vibrational average calculated for the $\mathrm{N}_{2} 3 \sigma_{\mathrm{g}}$ molecular orbital. The molecule was kept in a fixed orientation with respect to the photon and photoelectron wave vectors, but the $\mathrm{N}-\mathrm{N}$ bond was allowed to stretch and contract, as described in the Computational Details section. Figure 6b shows that, at high photon energies, the oscillations in the photoionization cross section are completely damped, while at low photon energies, they retain a relatively large amplitude. The reason for this is the fact that the photoelectron kinetic energy depends on the squared magnitude of the photoelectron wave vector. Small changes in $k_{\mathrm{e}}$ from its "ground state" value result in shifts of the order of tens of electronvolts at low photon energies, but the shifts are of the order of hundreds of electronvolts in the high photon energy region.

Besides molecular vibrations, another damping mechanism is represented by molecular rotations. To model the influence of rotations on the photoionization cross section, we rotated independently the photon and photoelectron wave vectors to directions generated via the Lebedev quadrature, as described in the Computational Details section. The averaged ND cross section obtained for the $3 \sigma_{\mathrm{g}} \mathrm{MO}$ of the $\mathrm{N}_{2}$ molecule is shown as a function of photon energy in Figure $6 \mathrm{c}$. As it can be seen from the figure, in this case the oscillations have almost completely vanished. Note that the rotationally averaged cross section is approximatively three times smaller than the vibrationally averaged value. This is due to the fact that the rotationally averaged cross section includes an average over possible photoelectron directions, not included in the fixed computational setup.

The results in Figure 6 show that the minimum values of the cross section of the valence electron photoemisson, that appear at regular intervals shown in Figure 6a, should be possible to detect experimentally. They are detectable for $\mathrm{N}_{2}$ and in fact for any diatomic molecule, or molecular system that can have extinction effects resulting from eqs 8 and 9. However, an experimental difficulty one would have to overcome is naturally the effect of rotations and vibrations that smooth out the minima (Figure 6b,c).

The last mechanism for damping out minima of the cross section of valence states is related to solid state measurements. Here the photoionized system is an ordered crystal, with valence states described by Bloch states. To model this situation for a cluster calculation, we have constructed a "poor man's" variant of a periodic structure and a Bloch state, as described in the Computational Details section. Figure 7 shows the results obtained for one of the valence molecular orbitals of the $\left[\mathrm{Ni}\left(\mathrm{H}_{2} \mathrm{O}\right)_{6}\right]^{2+}$ complex, which represents a molecular model for the $\mathrm{NiO}$ crystal. Note that in this complex the $\mathrm{Ni}$ atom sits in an octahedral cage of $\mathrm{O}$ atoms precisely analogous to $\mathrm{Ni}$ atoms in bulk $\mathrm{NiO}$. Also the nominal charge of the $\mathrm{Ni}$ and $\mathrm{O}$ atoms are the same in the two models. Figure 7a depicts 
the ND cross section as a function of photon energy calculated in a fixed setup, depicted in the inset, while Figure $7 \mathrm{~b}$ depicts the cross section obtained after k-point summation. The cross section in Figure 7a, which may be viewed as the cross section at the $\Gamma$ point, presents a large number of minima related to the wave-like nature of the photon and photoelectron. A kpoint summation performed over the full interval $[-2 \pi / a, 2 \pi / a]$ leads to an almost complete suppression of the oscillatory behavior. If the summation is performed on a smaller interval, the oscillations remain present but are damped. This would correspond to a metallic situation, where some of the bands cross the Fermi level.

ND Corrections. Tables 3, 4, and 5 list the maximum ND corrections calculated for the highest occupied molecular

Table 3. Maximum ND Corrections for the HOMO Orbital of the Diatomic Molecules (a)-(f) from Figure 1 Computed Using Rotationally Averaged Cross Sections (3rd Column, $\left.\Delta_{\mathrm{ND}}\right)$ and Using Differential Cross Sections at the Magic Angle (4th Column, $\Delta_{\mathrm{ND}}^{\mathrm{MA}}$ )

$\begin{array}{cccc}\text { molecule } & \hbar \omega \text { range }(\mathrm{keV}) & \Delta_{\mathrm{ND}}(\%) & \Delta_{\mathrm{ND}}^{\mathrm{MA}}(\%) \\ \mathrm{N}_{2} & <1 & 0.6 & 15 \\ & 1-5 & 3 & 28 \\ \mathrm{O}_{2} & 5-12 & 16 & 41 \\ & <1 & 0.4 & 13 \\ & 1-5 & 6 & 31 \\ \mathrm{~F}_{2} & 5-10 & 12 & 43 \\ & <1 & 0.6 & 12 \\ & 1-5 & 11 & 27 \\ \mathrm{Cl}_{2} & 5-12 & 9 & 43 \\ & <1 & 0.6 & 8 \\ & 1-5 & 6 & 27 \\ \mathrm{HF} & 5-12 & 13 & 42 \\ & <1 & 0.3 & 12 \\ & 1-5 & 3 & 30 \\ \mathrm{CO} & 5-12 & 7 & 43 \\ & <1 & 0.5 & 13 \\ & 1-5 & 3 & 25 \\ & 5-12 & 6 & 39\end{array}$

Table 4. Maximum ND Corrections for the HOMO Orbital of the Molecules $(\mathrm{g})-(\mathrm{k})$ from Figure 1, Calculated Using Rotationally Averaged Cross Sections (3rd Column, $\Delta_{\mathrm{ND}}$ ) and Using Differential Cross Sections at the Magic Angle (4th column, $\Delta_{\mathrm{ND}}^{\mathrm{MA}}$ )

$\begin{array}{cccc}\text { molecule } & \hbar \omega \text { range }(\mathrm{keV}) & \Delta_{\mathrm{ND}}(\%) & \Delta_{\mathrm{ND}}^{\mathrm{MA}}(\%) \\ \mathrm{H}_{2} \mathrm{O} & <1 & 0.4 & 13 \\ & 1-5 & 3 & 31 \\ & 5-12 & 7 & 44 \\ \mathrm{NH}_{3} & <1 & 0.3 & 12 \\ & 1-5 & 2 & 27 \\ & 5-12 & 5 & 38 \\ \mathrm{CH}_{4} & <1 & 0.7 & 16 \\ & 1-5 & 4 & 33 \\ & 5-12 & 10 & 43 \\ \mathrm{CH}_{2} \mathrm{O} & <1 & 0.6 & 13 \\ & 1-5 & 4 & 30 \\ & 5-12 & 8 & 44 \\ \mathrm{C}_{2} \mathrm{H}_{4} & <1 & 0.3 & 13 \\ & 1-5 & 4 & 35 \\ & 5-12 & 8 & 45\end{array}$

Table 5. Maximum ND Corrections for the HOMO Orbital of the Molecules (1)-(q) from Figure 1, Calculated Using Rotationally Averaged Cross Sections (3rd Column, $\Delta_{\mathrm{ND}}$ ) and Using Differential Cross Sections at the Magic Angle (4th Column, $\Delta_{\mathrm{ND}}^{\mathrm{MA}}$ )

$\begin{array}{cccc}\text { molecule } & \hbar \omega \text { range }(\mathrm{keV}) & \Delta_{\mathrm{ND}}(\%) & \Delta_{\mathrm{ND}}^{\mathrm{MA}}(\%) \\ \mathrm{C}_{6} \mathrm{H}_{6} & <1 & 2 & 13 \\ & 1-5 & 9 & 34 \\ {\left[\mathrm{Ni}\left(\mathrm{H}_{2} \mathrm{O}\right)_{6}\right]^{2+}} & 5-12 & 13 & 46 \\ & <1 & 0.5 & 13 \\ 3 \mathrm{HT} & 1-5 & 9 & 37 \\ & 5-12 & 11 & 44 \\ & <1 & 0.6 & 12 \\ \mathrm{C}_{60} & 1-5 & 5 & 28 \\ & 5-12 & 9 & 43 \\ \mathrm{ZnP} & <1 & 3 & 14 \\ & 1-5 & 12 & 37 \\ & 5-12 & 14 & 47 \\ \mathrm{NiPc} & <1 & 4 & 15 \\ & 1-5 & 9 & 26 \\ & 5-12 & 7 & 38 \\ & <1 & 5 & 19 \\ & 1-5 & 19 & 34 \\ & 5-12 & 17 & 47 \\ & & & \end{array}$

orbital (HOMO) of all molecules in Figure 1 on three photon energy intervals, below $1 \mathrm{keV}$, between $1-5 \mathrm{keV}$, and between 5-12 keV. Two corrections have been calculated, one using the roationally averaged ND and ED cross sections and one using differential cross sections computed at the magic angle $\theta$ $=54.7^{\circ}$.

Although rotational averaging strongly reduces the oscillatory behavior previously discussed, the oscillations are in fact not completely removed and they can be observed experimentally, as for example in refs 85,84 , and 36.

Korica et al. ${ }^{84,85}$ observed relative intensity oscillations as a function of photon energy in the valence PE spectra of the $\mathrm{C}_{60}$ and $\mathrm{C}_{70}$ fullerenes. The observed oscillatory behavior was related to the fact that the wavelength of the emitted photoelectron matched the diameter of the fullerene cage. This is the same mechanism as described in the Photoionization of Valence Levels section for the $\mathrm{N}_{2}$ molecule. A comparison between the measured data for $\mathrm{C}_{60}$ from ref 85 and the corresponding values computed using ND differential cross sections as a function of photon energy is shown in the Supporting Information, Figure S1. Although the photon energy is low and the plane wave approximation for the final state is less reliable, the theoretical and experimental results compare quite well, suggesting that the experimentally observed oscillations are mainly due to the effect discussed in the Photoionization of Valence Levels section. However, depending on the system and the type of measurements, additional effects, such as transitions between vibrational levels, ${ }^{36}$ or multiple scattering ${ }^{79}$ can also play a role.

While the ED cross sections have minima due only to the wave-like nature of the photoelectron, the ND cross sections are influenced by both the photon and the photoelectron. Besides the magnitude of the two wave vectors, also their relative directions will affect the photon energy position of the cross section minima. Compared to atomic photoionization, where the magnitude of the ND corrections was influenced only by (1) the magnitude and (2) the direction of the 
resulting vector $\mathbf{K}=\mathbf{k}_{\mathrm{e}}-\mathbf{k},{ }^{53}$ ND corrections for valence molecular orbitals are additionally affected by (3) the shift in photon energy position of the cross section minima. Due to the remaining oscillatory behavior in the cross sections after rotational averaging, the ND corrections themselves display oscillations, as depicted in Figure 8 for the $3 \sigma_{\mathrm{g}}$ and $1 \pi_{\mathrm{u}}$ orbitals

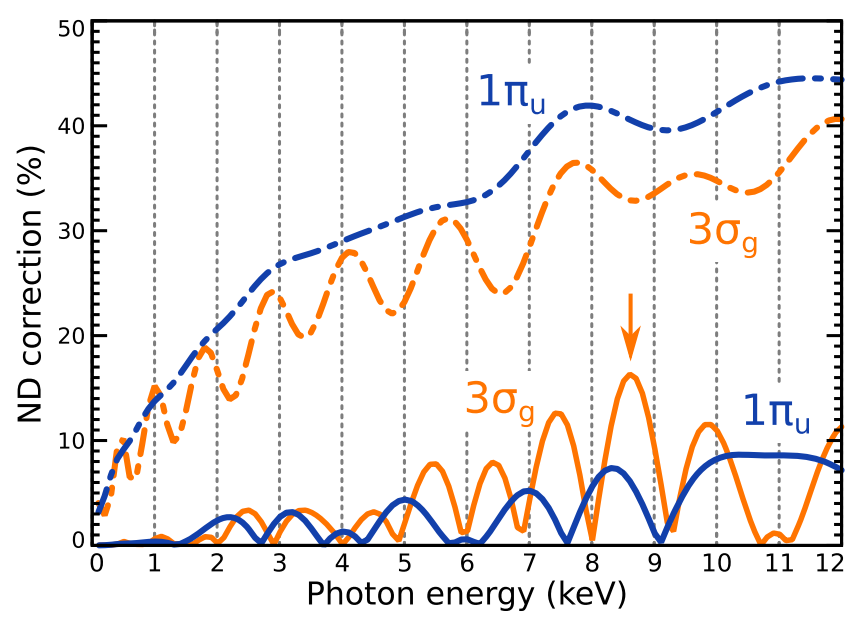

Figure 8. Calculated ND corrections to the rotationally averaged photoionization cross sections (solid line) and to the differential cross sections (dashed-dotted lines) of the $3 \sigma_{\mathrm{g}}$ (in orange) and $1 \pi_{\mathrm{u}}$ (in blue) molecular orbitals of the $\mathrm{N}_{2}$ molecule. The differential cross sections are computed at the magic angle $\theta=54.7^{\circ}$.

of the $\mathrm{N}_{2}$ molecule. Additionally, because of the mathematical relation between the magnitude of the photoelectron wave vector and the photoelectron kinetic energy, as discussed in the Photoionization of Valence Levels section, the largest ND corrections may not necessarily be observed at the highest photon energy. At low photon energies $(<1 \mathrm{keV})$, where the magnitude of the photon wave vector is small, the ED and ND cross sections are very similar. At high photon energies, where the amplitudes of the oscillations are strongly reduced, the ND corrections are less affected by the shift in photon energy position of the cross section minima. However, in a midrange photon energy region, where the photon wave vector is large enough but where the magnitude of the photoelectron wave vector is small enough, all three mechanisms discussed above will give rise to differences between the ED and the ND cross sections. The photon energy range where this happens depends on the particular system and molecular orbital.

This is to some extent illustrated in Tables 3 and 5, where the maximum ND corrections for the rotationally averaged cross sections of $\mathrm{F}_{2}$ and $\mathrm{NiPc}$ are obtained mid-range. On the other hand, this effect is clearly visible in Figure 8, where the maximum correction obtained for the rotationally averaged cross section of the $3 \sigma_{\mathrm{g}}$ orbital is obtained at approximately 8.5 $\mathrm{keV}$ (marked with an arrow in the figure). It is expected that this effect would be even more pronounced in the absence of damping mechanisms, as, for example, for fixed-in-space molecules ${ }^{86}$ or molecules deposited on surfaces.

The rotationally averaged ND corrections are generally small. At low photon energies, below $1 \mathrm{keV}$, the corrections are smaller than $1 \%$ for all systems, except in the case of the larger molecules, with delocalized HOMO orbitals, i.e., benzene $\left(\mathrm{C}_{6} \mathrm{H}_{6}, 2 \%\right), \mathrm{C}_{60}$ (3\%), $\mathrm{ZnP}(4 \%)$, and NiPc (5\%). The corrections increase with photon energy reaching up to $19 \%$ in the case of NiPc.
The ND corrections obtained using differential cross sections at the magic angle are comparatively large for all systems. Corrections to the differential photoionization cross sections have been calculated before by Seabra et al. ${ }^{64}$ for HF, $\mathrm{H}_{2} \mathrm{O}, \mathrm{NH}_{3}$, and $\mathrm{CH}_{4}$. When taking into account the different basis set used, 6-311G(d,p) (Seabra and co-workers) vs UGBS (this work), we reproduce the results obtained in ref 64. This is illustrated in the Supporting Information, Figure S2, in the case of the HOMO-1 orbital of $\mathrm{NH}_{3}$, where the ND correction to the differential cross section has been computed using the UGBS and the $6-311 \mathrm{G}(\mathrm{d}, \mathrm{p})$ basis sets as a function of photon energy.

The ND corrections calculated for differential cross sections at a fixed angle $\theta=54.7^{\circ}$ between the photon and photoelectron wave vectors present oscillations as a function of photon energy related to the cross section minima, as discussed before. This is clearly visible in the correction to the differential cross section of the $3 \sigma_{\mathrm{g}}$ orbital of $\mathrm{N}_{2}$, shown in Figure 8 . In contrast to the case of the rotationally averaged cross sections, the corrections for the differential cross section steadily increase with photon energy in a similar manner for all systems, and the main difference between molecules and orbitals is due to the type of oscillatory behavior-see, for example, the difference between $3 \sigma_{\mathrm{g}}$ and $1 \pi_{\mathrm{u}}$ in Figure 8 . The fact that the ND corrections to the differential cross sections are much larger than the corrections to the rotationally averaged ones suggests that ND effects will especially affect angle-resolved PE spectra, even at photon energies as low as 1 $\mathrm{keV}$. Once again, ND corrections are expected to be even larger for systems where the damping mechanisms discussed in the Photoionization of Valence Levels section are missing, such as fixed-in-space molecules or molecules adsorbed on surfaces.

\section{CONCLUSIONS}

We have applied a methodology to compute molecular photoionization cross sections beyond the electric dipole approximation using the full field operator in combination with GTOs and PWs to describe the initial and final states, respectively. The methodology is computationally inexpensive and can be easily applied to large systems, such as the $\mathrm{C}_{60}$ fullerene or transition-metal phthalocyanines. The molecular photoionization cross sections display oscillations related to the wave-like nature of the incoming photon, as well as of the outgoing photoelectron. The oscillatory behavior vanishes when the bonding and antibonding molecular orbitals are degenerate and their corresponding cross sections can be summed up. This is the case for core orbitals, but not for valence orbitals, where the bonding and antibonding counterparts are either nondegenerate or partly filled. However, the oscillatory behavior of the cross sections is damped by rotational and vibrational averaging (gas-phase and liquid), or by k-point summation (solid state). The ND corrections for the localized core orbitals are very similar to the atomic case, reaching up to $5-10 \%$ at $12 \mathrm{keV}$, depending on the orbital type. The ND corrections to rotationally averaged cross sections of valence orbitals reach up to $\sim 20 \%$, as in the case of the HOMO orbital of NiPc. Due to the oscillatory behavior of the cross sections, above a certain photon energy threshold, the maximum correction is not necessarily obtained at the highest photon energy. ND corrections become much larger when angle-resolved calculations are performed. Keeping the angle between the photon and photoelectron wave vectors fixed at $\theta$ 
$=54.7^{\circ}$ results in corrections of approximately $50 \%$ in the cases of $\mathrm{C}_{60}$ and $\mathrm{NiPc}$ at photon energies between $5-12 \mathrm{keV}$.

In this investigation we find that the cross section of both core and valence orbitals can reach distinct minimum values for specific energies, which should be possible to detect experimentally. We have pointed to some observations in the literature, and we show here that both rotational and vibrational effects diminish these conspicuous minimum values. Hence an experiment that could suppress these effects, either by reaching low temperature or by studies of chemisorbed molecular species, would be highly interesting. Here angle resolved photoemission spectroscopy (ARPES) might also offer an interesting experimental way to detect distinct minima of the cross sections, since in these experiments smearing of the minima due to a summation of different k-points (Figure $7 \mathrm{~b}$ ) is avoided. In the case of core spectroscopy these minimum values would have to be detected by means of two-photon experiments, where one photon first kicks out an electron, creating an open shell, and then a second photon is used to detect the cross section minima of the remaining occupied core orbital.

\section{ASSOCIATED CONTENT}

\section{S Supporting Information}

The Supporting Information is available free of charge on the ACS Publications website at DOI: 10.1021/acs.jctc.9b00470.

(a) Ratio between differential ND photoionization cross sections of the HOMO and HOMO-1 orbitals of $\mathrm{C}_{60}$ calculated at the magic angle between the photon and photoelectron wave vectors, compared to experimental $\mathrm{PE}$ data from ref 85 and (b) ND corrections calculated for the HOMO-1 orbital of $\mathrm{NH}_{3}$ using two different basis sets, UGBS and, respectively, 6-311G(d,p), in the photon energy range between $10 \mathrm{eV}$ and $5 \mathrm{keV}$, shown in comparison to the results from ref 64 for the same molecule (PDF)

\section{AUTHOR INFORMATION}

\section{Corresponding Author}

*(I.E.B.) E-mail: iubr@kth.se.

\section{ORCID}

Iulia Emilia Brumboiu: 0000-0003-1671-8298

Patrick Norman: 0000-0002-1191-4954

\section{Funding}

Financial support from the Knut and Alice Wallenberg Foundation (Grant No. KAW-2013.0020) and the Swedish Research Council (Grant No. 621-2014-4646 and Grant No. 2017-06419) is acknowledged. The computations were performed on resources provided by the Swedish National Infrastructure for Computing (SNIC) at NSC and HPC2N. O.E. also acknowledges support from the Swedish Research Council (VR) and eSSENCE.

\section{Notes}

The authors declare no competing financial interest.

\section{ACKNOWLEDGMENTS}

I.E.B. is very grateful to Faris Gel'mukhanov, Nanna Holmgaard List, Barbara Brena, and Igor Di Marco for all the helpful discussions.

\section{REFERENCES}

(1) Green, J. C. Variable photon energy photoelectron spectroscopy of transition metal molecules. Acc. Chem. Res. 1994, 27, 131-137.

(2) Green, J. C.; Decleva, P. Photoionization cross-sections: a guide to electronic structure. Coord. Chem. Rev. 2005, 249, 209-228.

(3) Hammar, H.; Fransson, J. Time-dependent spin and transport properties of a single-molecule magnet in a tunnel junction. Phys. Rev. B: Condens. Matter Mater. Phys. 2016, 94, 054311.

(4) Saygun, T.; Bylin, J.; Hammar, H.; Fransson, J. Voltage-induced switching dynamics of a coupled spin pair in a molecular junction. Nano Lett. 2016, 16, 2824-2829.

(5) Kronik, L.; Kümmel, S. In First principles approaches to spectroscopic properties of complex materials; De Valentin, C., Botti, S., Cococcioni, M., Eds.; Springer: Berlin, Heidelberg, 2014; pp 137191.

(6) Kümmel, S.; Kronik, L. Orbital-dependent density functionals: theory and applications. Rev. Mod. Phys. 2008, 80, 3.

(7) Egger, D. A.; Weissman, S.; Refaely-Abramson, S.; Sharifzadeh, S.; Dauth, M.; Baer, R.; Kümmel, S.; Neaton, J. B.; Zojer, E.; Kronik, L. Outer-valence electron spectra of prototypical aromatic heterocycles from an optimally tuned range-separated hybrid functional. $J$. Chem. Theory Comput. 2014, 10, 1934-1952.

(8) Brumboiu, I. E.; Prokopiou, G.; Kronik, L.; Brena, B. Valence electronic structure of cobalt phthalocyanine from an optimally tuned range-separated hybrid functional. J. Chem. Phys. 2017, 147, 044301.

(9) Liu, Z.-F.; Egger, D. A.; Refaely-Abramson, S.; Kronik, L.; Neaton, J. B. Energy level alignment at molecule-metal interfaces from an optimally tuned range-separated hybrid functional. J. Chem. Phys. 2017, 146, 092326.

(10) Guo, C.; Sarkar, S.; Refaely-Abramson, S.; Egger, D. A.; Bendikov, T.; Yonezawa, K.; Suda, Y.; Yamaguchi, T.; Pecht, I.; Kera, S.; et al. Electronic structure of dipeptides in the gas-phase and as an adsorbed monolayer. Phys. Chem. Chem. Phys. 2018, 20, 6860-6867.

(11) Marom, N.; Caruso, F.; Ren, X.; Hofmann, O. T.; Körzdörfer, T.; Chelikowsky, J. R.; Rubio, A.; Scheffler, M.; Rinke, P. Benchmark of GW methods for azabenzenes. Phys. Rev. B: Condens. Matter Mater. Phys. 2012, 86, 245127.

(12) Körzdörfer, T.; Marom, N. Strategy for finding a reliable starting point for $G_{0} W_{0}$ demonstrated for molecules. Phys. Rev. B: Condens. Matter Mater. Phys. 2012, 86, 041110.

(13) Marom, N.; Kronik, L. Density functional theory of transition metal phthalocyanines, I: Electronic structure of NiPc and CoPc-selfinteraction effects. Appl. Phys. A: Mater. Sci. Process. 2009, 95, 159163.

(14) Marom, N.; Kronik, L. Density functional theory of transition metal phthalocyanines, II: electronic structure of $\mathrm{MnPc}$ and $\mathrm{FePc}-$ symmetry and symmetry breaking. Appl. Phys. A: Mater. Sci. Process. 2009, 95, 165-172.

(15) Zhang, T.; Brumboiu, I. E.; Grazioli, C.; Guarnaccio, A.; Coreno, M.; de Simone, M.; Santagata, A.; Rensmo, H.; Brena, B.; Lanzilotto, V.; et al. Lone-Pair delocalization effects within electron donor molecules: the case of triphenylamine and its thiophene-analog. J. Phys. Chem. C 2018, 122, 17706-17717.

(16) Brumboiu, I. E.; Haldar, S.; Lüder, J.; Eriksson, O.; Herper, H. C.; Brena, B.; Sanyal, B. Influence of electron correlation on the electronic structure and magnetism of transition-metal phthalocyanines. J. Chem. Theory Comput. 2016, 12, 1772-1785.

(17) Metzner, W.; Vollhardt, D. Correlated lattice fermions in $\mathrm{d}=\infty$ dimensions. Phys. Rev. Lett. 1989, 62, 324-327.

(18) Georges, A.; Kotliar, G.; Krauth, W.; Rozenberg, M. J. Dynamical mean-field theory of strongly correlated fermion systems and the limit of infinite dimensions. Rev. Mod. Phys. 1996, 68, 13125 .

(19) Brumboiu, I. E.; Totani, R.; de Simone, M.; Coreno, M.; Grazioli, C.; Lozzi, L.; Herper, H. C.; Sanyal, B.; Eriksson, O.; Puglia, C.; et al. Elucidating the $3 \mathrm{~d}$ electronic configuration in manganese phthalocyanine. J. Phys. Chem. A 2014, 118, 927-932.

(20) Brena, B.; Puglia, C.; de Simone, M.; Coreno, M.; Tarafder, K.; Feyer, V.; Banerjee, R.; Göthelid, E.; Sanyal, B.; Oppeneer, P. M.; 
et al. Valence-band electronic structure of iron phthalocyanine: An experimental and theoretical photoelectron spectroscopy study. J. Chem. Phys. 2011, 134, 074312.

(21) Zhang, T.; Brumboiu, I. E.; Lanzilotto, V.; Lüder, J.; Grazioli, C.; Giangrisostomi, E.; Ovsyannikov, R.; Sassa, Y.; Bidermane, I.; Stupar, M.; et al. Conclusively addressing the CoPc electronic structure: a joint gas-phase and solid-state photoemission and absorption spectroscopy study. J. Phys. Chem. C 2017, 121, 2637226378 .

(22) Bidermane, I.; Brumboiu, I. E.; Totani, R.; Grazioli, C.; Shariati-Nilsson, M. N.; Herper, H. C.; Eriksson, O.; Sanyal, B.; Ressel, B.; de Simone, M.; et al. Atomic contributions to the valence band photoelectron spectra of metal-free, iron and manganese phthalocyanines. J. Electron Spectrosc. Relat. Phenom. 2015, 205, 9297.

(23) Panda, S. K.; Pal, B.; Mandal, S.; Gorgoi, M.; Das, S.; Sarkar, I.; Drube, W.; Sun, W.; Di Marco, I.; Lindblad, A.; et al. High photon energy spectroscopy of $\mathrm{NiO}$ : experiment and theory. Phys. Rev. B: Condens. Matter Mater. Phys. 2016, 93, 235138.

(24) Paul, S.; Iuşan, D.; Thunström, P.; Kvashnin, Y. O.; Hellsvik, J.; Pereiro, M.; Delin, A.; Knut, R.; Phuyal, D.; Lindblad, A.; et al. Investigation of the spectral properties and magnetism of $\mathrm{BiFeO}_{3}$ by dynamical mean-field theory. Phys. Rev. B: Condens. Matter Mater. Phys. 2018, 97, 125120.

(25) Yeh, J.; Lindau, I. Atomic subshell photoionization cross sections and asymmetry parameters: $1 \leq \mathrm{Z} 1 \leq 03$. At. Data Nucl. Data Tables 1985, 32, 1-155.

(26) Yeh, J. Atomic calculation of photoionization cross-sections and asymmetry parameters; Gordon \& Breach Science, Publishers: 1993.

(27) Atomic calculation of photoionization cross-sections and asymmetry parameters. https://vuo.elettra.eu/services/elements/ WebElements.html (accessed Sep. 13, 2018; archived by WebCite at http://www.webcitation.org/72OwdTOR5).

(28) Gozem, S.; Gunina, A. O.; Ichino, T.; Osborn, D. L.; Stanton, J. F.; Krylov, A. I. Photoelectron wave function in photoionization: plane wave or Coulomb wave? J. Phys. Chem. Lett. 2015, 6, 45324540.

(29) Seabra, G. M.; Kaplan, I. G.; Zakrzewski, V. G.; Ortiz, J. V. Electron propagator theory calculations of molecular photoionization cross sections: the first-row hydrides. J. Chem. Phys. 2004, 121, 41434155.

(30) Melania Oana, C.; Krylov, A. I. Dyson orbitals for ionization from the ground and electronically excited states within equation-ofmotion coupled-cluster formalism: theory, implementation, and examples. J. Chem. Phys. 2007, 127, 234106.

(31) Oana, C. M.; Krylov, A. I. Cross sections and photoelectron angular distributions in photodetachment from negative ions using equation-of-motion coupled-cluster Dyson orbitals. J. Chem. Phys. 2009, 131, 124114.

(32) Ponzi, A.; Sapunar, M.; Angeli, C.; Cimiraglia, R.; Došlić, N.; Decleva, P. Photoionization of furan from the ground and excited electronic states. J. Chem. Phys. 2016, 144, 084307.

(33) Stener, M.; Decleva, P. Time-dependent density functional calculations of molecular photoionization cross sections: $\mathrm{N}_{2}$ and. $\mathrm{PH}_{3}$. J. Chem. Phys. 2000, 112, 10871-10879.

(34) Stener, M.; Fronzoni, G.; Decleva, P. Time-dependent densityfunctional theory for molecular photoionization with noniterative algorithm and multicenter B-spline basis set: $\mathrm{CS}_{2}$ and $\mathrm{C}_{6} \mathrm{H}_{6}$ case studies. J. Chem. Phys. 2005, 122, 234301.

(35) Martín, F. Ionization and dissociation using B-splines: photoionization of the hydrogen molecule. J. Phys. B: At., Mol. Opt. Phys. 1999, 32, R197.

(36) Canton, S. E.; Plésiat, E.; Bozek, J. D.; Rude, B. S.; Decleva, P.; Martín, F. Direct observation of Young's double-slit interferences in vibrationally resolved photoionization of diatomic molecules. Proc. Natl. Acad. Sci. U. S. A. 2011, 108, 7302.

(37) Minár, J. In DMFT at 25: infinite dimensions, modeling and simulation; Pavarini, E., Koch, E., Vollhardt, D., Lichtenstein, A., Eds.; Verlag des Forschungszentrum Jülich: 2014; Vol. 4, Chapter 13.
(38) Braun, J.; Minár, J.; Ebert, H. In Hard X-ray photoelectron spectroscopy (HAXPES); Woicik, J., Ed.; Springer International Publishing: 2016; pp 159-174.

(39) Keqi, A.; Gehlmann, M.; Conti, G.; Nemšák, S.; Rattanachata, A.; Minár, J.; Plucinski, L.; Rault, J. E.; Rueff, J. P.; Scarpulla, M.; et al. Electronic structure of the dilute magnetic semiconductor $\mathrm{Ga}_{1-x} \mathrm{Mn}_{x} \mathrm{P}$ from hard $\mathrm{x}$-ray photoelectron spectroscopy and angle-resolved photoemission. Phys. Rev. B: Condens. Matter Mater. Phys. 2018, 97, 155149 .

(40) Veis, M.; Minár, J.; Steciuk, G.; Palatinus, L.; Rinaldi, C.; Cantoni, M.; Kriegner, D.; Tikuišis, K. K.; Hamrle, J.; Zahradník, M.; et al. Band structure of CuMnAs probed by optical and photoemission spectroscopy. Phys. Rev. B: Condens. Matter Mater. Phys. 2018, 97, 125109.

(41) Gray, A. X.; Papp, C.; Ueda, S.; Balke, B.; Yamashita, Y.; Plucinski, L.; Minár, J.; Braun, J.; Ylvisaker, E. R.; Schneider, C. M.; et al. Probing bulk electronic structure with hard X-ray angle-resolved photoemission. Nat. Mater. 2011, 10, 759.

(42) Minár, J.; Ebert, H.; Ghiringhelli, G.; Tjernberg, O.; Brookes, N. B.; Tjeng, L. H. Theoretical description of the Fano effect in the angle-integrated valence-band photoemission of paramagnetic solids. Phys. Rev. B: Condens. Matter Mater. Phys. 2001, 63, 144421.

(43) Derevianko, A.; Johnson, W. R.; Cheng, K. T. Non-dipole effects in photoelectron angular distributions for rare gas atoms. At. Data Nucl. Data Tables 1999, 73, 153-211.

(44) Cooper, J. W. Photoelectron-angular-distribution parameters for rare-gas subshells. Phys. Rev. A: At., Mol., Opt. Phys. 1993, 47, $1841-1851$

(45) Bechler, A.; Pratt, R. H. Higher retardation and multipole corrections to the dipole angular distribution of $1 \mathrm{~s}$ photoelectrons at low energies. Phys. Rev. A: At., Mol., Opt. Phys. 1989, 39, 1774-179.

(46) Bechler, A.; Pratt, R. H. Higher multipole and retardation corrections to the dipole angular distributions of L-shell photoelectrons ejected by polarized photons. Phys. Rev. A: At., Mol., Opt. Phys. 1990, 42, 6400-6413.

(47) Nefedov, V. I.; Yarzhemsky, V. G.; Nefedova, I. S.; Trzhaskovskaya, M. B.; Band, I. M. The influence of non-dipolar transitions on the angular photoelectron distribution. J. Electron Spectrosc. Relat. Phenom. 2000, 107, 123-130.

(48) Amusia, M. Y.; Baltenkov, A. S.; Chernysheva, L. V.; Felfli, Z.; Msezane, A. Z. Nondipole parameters in angular distributions of electrons in photoionization of noble-gas atoms. Phys. Rev. A: At., Mol., Opt. Phys. 2001, 63, 052506.

(49) Dolmatov, V. K.; Manson, S. T. Large nondipole parameters with strong correlation effects in photoelectron angular distributions at kilo-electron-volt energies. Phys. Rev. A: At., Mol., Opt. Phys. 2001, 63, 022704.

(50) Derevianko, A.; Hemmers, O.; Oblad, S.; Glans, P.; Wang, H.; Whitfield, S. B.; Wehlitz, R.; Sellin, I. A.; Johnson, W. R.; Lindle, D. $\mathrm{W}$. Electric-octupole and pure-electric-quadrupole effects in soft-X-ray photoemission. Phys. Rev. Lett. 2000, 84, 2116-2119.

(51) Simon, M.; Piancastelli, M. N.; Lindle, D. W. In Hard X-ray photoelectron spectroscopy (HAXPES); Woicik, J., Ed.; Springer International Publishing: 2016; pp 65-110.

(52) Demekhin, P. V. On the breakdown of the electric dipole approximation for hard $\mathrm{x}$-ray photoionization cross sections. J. Phys. B: At., Mol. Opt. Phys. 2014, 47, 025602.

(53) Brumboiu, I. E.; Eriksson, O.; Norman, P. Atomic photoionization cross sections beyond the electric dipole approximation. J. Chem. Phys. 2019, 150, 044306.

(54) Zimmermann, B.; McKoy, V.; Southworth, S.; Kanter, E.; Krässig, B.; Wehlitz, R. Dipole and nondipole photoionization of molecular hydrogen. Phys. Rev. A: At., Mol., Opt. Phys. 2015, 91, 053410 .

(55) Guillemin, R.; Hemmers, O.; Lindle, D. W.; Manson, S. T. Experimental investigation of nondipole effects in photoemission at the advanced light source. Radiat. Phys. Chem. 2006, 75, 2258-2274.

(56) Hosaka, K.; Adachi, J.; Golovin, A. V.; Takahashi, M.; Teramoto, T.; Watanabe, N.; Jahnke, T.; Weber, Th.; Schoffler, M.; 
Schmidt, L.; et al. Nondipole effects in the angular distribution of photoelectrons from the $\mathrm{C} \mathrm{K}$ shell of the CO molecule. Phys. Rev. A: At., Mol., Opt. Phys. 2006, 73, 022716.

(57) Southworth, S.; Dunford, R.; Kanter, E.; Krässig, B.; Young, L.; LaJohn, L.; Pratt, R. Nondipole asymmetries of K-shell photoelectrons of $\mathrm{Kr}, \mathrm{Br} 2$, and BrCF3. Radiat. Phys. Chem. 2006, 75, 1574-1577.

(58) Hosaka, K.; Adachi, J.; Golovin, A.; Takahashi, M.; Teramoto, T.; Watanabe, N.; Yagishita, A.; Semenov, S.; Cherepkov, N. Nondipole effects in the angular distribution of photoelectrons from the K-shell of $\mathrm{N}_{2}$ molecule. J. Phys. B: At., Mol. Opt. Phys. 2006, 39, L25.

(59) Toffoli, D.; Decleva, P. A multicentric approach to the calculation of nondipolar effects in molecular photoemission. J. Chem. Phys. 2008, 128, 234101.

(60) Stener, M.; Toffoli, D.; Fronzoni, G.; Decleva, P. Recent advances in molecular photoionization by density functional theory based approaches. Theor. Chem. Acc. 2007, 117, 943-956.

(61) Toffoli, D.; Decleva, P. Photoelectron angular distributions beyond the dipole approximation: a computational study on the $\mathrm{N}_{2}$ molecule. J. Phys. B: At., Mol. Opt. Phys. 2006, 39, 2681-2691.

(62) Toffoli, D.; Decleva, P. Nondipolar effects in the photoionization dynamics of carbon tetrafluoride. Phys. Rev. A: At., Mol., Opt. Phys. 2008, 78, 063402.

(63) Toffoli, D.; Decleva, P. Strong oscillations in the nondipole corrections to the photoelectron angular distributions from $\mathrm{C}_{60}$. Phys. Rev. A: At., Mol., Opt. Phys. 2010, 81, 061201.

(64) Seabra, G. M.; Kaplan, I. G.; Ortiz, J. V. Molecular photoionization cross sections in electron propagator theory: angular distributions beyond the dipole approximation. J. Chem. Phys. 2005, $123,114105$.

(65) List, N. H.; Kauczor, J.; Saue, T.; Jensen, H. J. A.; Norman, P. Beyond the electric-dipole approximation: A formulation and implementation of molecular response theory for the description of absorption of electromagnetic field radiation. J. Chem. Phys. 2015, 142, 244111.

(66) List, N. H.; Saue, T.; Norman, P. Rotationally averaged linear absorption spectra beyond the electric-dipole approximation. Mol. Phys. 2017, 115, 63-74.

(67) Frisch, M. J.; Trucks, G. W.; Schlegel, H. B.; Scuseria, G. E.; Robb, M. A.; Cheeseman, J. R.; Scalmani, G.; Barone, V.; Mennucci, B.; Petersson, G. A.; et al. Gaussian 16, Revision A.03; Gaussian, Inc.: Wallingford, CT, 2016.

(68) Becke, A. D. A new mixing of Hartree-Fock and local densityfunctional theories. J. Chem. Phys. 1993, 98, 1372-1377.

(69) Silver, D. M.; Wilson, S.; Nieuwpoort, W. C. Universal basis sets and transferability of integrals. Int. J. Quantum Chem. 1978, 14, 635-639.

(70) Silver, D. M.; Nieuwpoort, W. C. Universal atomic basis sets. Chem. Phys. Lett. 1978, 57, 421-422.

(71) Mohallem, J. R.; Dreizler, R. M.; Trsic, M. A Griffin-HillWheeler version of the Hartree-Fock equations. Int. J. Quantum Chem. 1986, 30, 45-55.

(72) Mohallem, J. R.; Trsic, M. A universal Gaussian basis set for atoms $\mathrm{Li}$ through $\mathrm{Ne}$ based on a generator coordinate version of the Hartree-Fock equations. J. Chem. Phys. 1987, 86, 5043-5044.

(73) da Costa, H. F. M.; Trsic, M.; Mohallem, J. R. Universal gaussian and Slater-type basis sets for atoms $\mathrm{He}$ to Ar based on an integral version of the Hartree-Fock equations. Mol. Phys. 1987, 62, 91-95.

(74) da Silva, A. B. F.; Da Costa, H. F. M.; Trsic, M. Universal gaussian and Slater-type bases for atoms $\mathrm{H}$ to $\mathrm{Xe}$ based on the generator coordinate Hartree-Fock method: I. ground and certain low-lying excited states of the neutral atoms. Mol. Phys. 1989, 68, 433-445.

(75) Jorge, F. E.; De Castro, E. V. R.; Da Silva, A. B. F. A universal Gaussian basis set for atoms cerium through lawrencium generated with the generator coordinate Hartree-Fock method. J. Comput. Chem. 1997, 18, 1565-1569.

(76) Jorge, F. E.; De Castro, E. V. R.; Da Silva, A. B. F. Accurate universal Gaussian basis set for hydrogen through lanthanum generated with the generator coordinate Hartree-Fock method. Chem. Phys. 1997, 216, 317-321.

(77) de Castro, E. V. R.; Jorge, F. E. Accurate universal gaussian basis set for all atoms of the periodic table. J. Chem. Phys. 1998, 108, 5225-5229.

(78) Lebedev, V. I. Quadratures on a sphere. USSR Comput. Math. \& Math. Phys. 1976, 16, 10-24.

(79) Liu, X. J.; Cherepkov, N. A.; Semenov, S. K.; Kimberg, V.; Gel'mukhanov, F.; Prümper, G.; Lischke, T.; Tanaka, T.; Hoshino, M.; Tanaka, H.; et al. Young's double-slit experiment using core-level photoemission from $\mathrm{N}_{2}$ : revisiting Cohen-Fano's two-centre interference phenomenon. J. Phys. B: At., Mol. Opt. Phys. 2006, 39, 4801-4817.

(80) Cohen, H. D.; Fano, U. Interference in the photo-ionization of molecules. Phys. Rev. 1966, 150, 30-33.

(81) Söderström, J.; Mårtensson, N.; Travnikova, O.; Patanen, M.; Miron, C.; Sæthre, L. J.; Børve, K. J.; Rehr, J. J.; Kas, J. J.; Vila, F. D.; et al. Nonstoichiometric intensities in core photoelectron spectroscopy. Phys. Rev. Lett. 2012, 108, 193005.

(82) Ankudinov, A. L.; Ravel, B.; Rehr, J. J.; Conradson, S. D. Realspace multiple-scattering calculation and interpretation of $\mathrm{x}$-rayabsorption near-edge structure. Phys. Rev. B: Condens. Matter Mater. Phys. 1998, 58, 7565-7576.

(83) Rehr, J. J.; Albers, R. C. Theoretical approaches to x-ray absorption fine structure. Rev. Mod. Phys. 2000, 72, 621-654.

(84) Korica, S.; Reinköster, A.; Braune, M.; Viefhaus, J.; Rolles, D.; Langer, B.; Fronzoni, G.; Toffoli, D.; Stener, M.; Decleva, P.; et al. Partial photoionization cross sections of $\mathrm{C}_{60}$ and: $\mathrm{C}_{70}$ a gas versus adsorbed phase comparison. Surf. Sci. 2010, 604, 1940-1944.

(85) Korica, S.; Rolles, D.; Reinköster, A.; Langer, B.; Viefhaus, J.; Cvejanović, S.; Becker, U. Partial cross sections and angular distributions of resonant and nonresonant valence photoemission of $\mathrm{C}_{60}$. Phys. Rev. A: At., Mol., Opt. Phys. 2005, 71, 013203.

(86) Zimmermann, B.; Rolles, D.; Langer, B.; Hentges, R.; Braune, M.; Cvejanovic, S.; Geßner, O.; Heiser, F.; Korica, S.; Lischke, T.; et al. Localization and loss of coherence in molecular double-slit experiments. Nat. Phys. 2008, 4, 649-655. 